\title{
Entrevista a la Dra. Dulce María Núñez
}

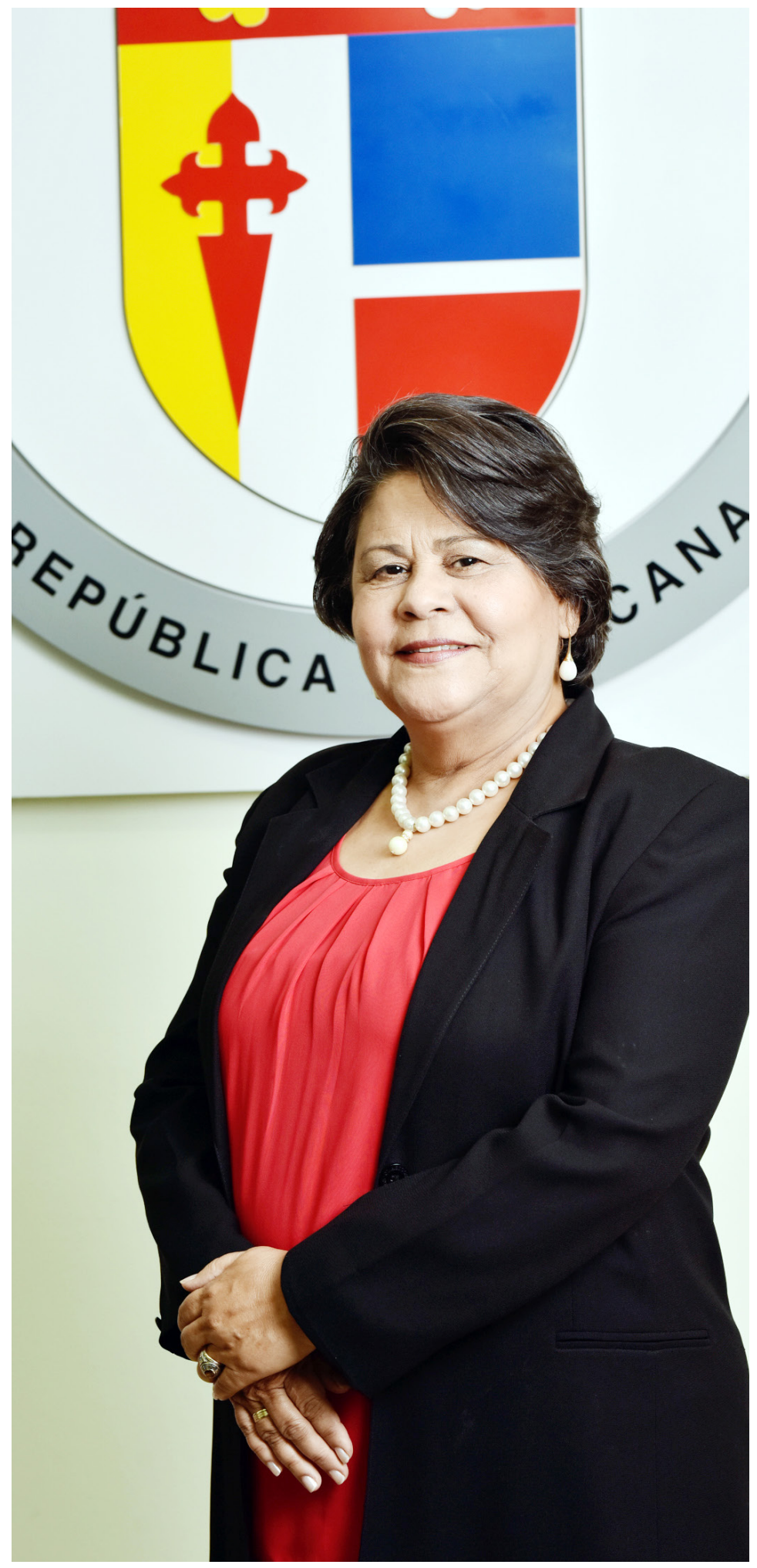

¿Desde cuándo inicia su labor en la PUCMM?

Inicié mi labor como auxiliar de biblioteca en el año 1986 y he estado allí por 32 años al servicio de los usuarios con el objetivo de acercar el conocimiento a ellos.

¿Cuál es su carrera inicial y cómo logró armonizar su disciplina con el trabajo de la Biblioteca?

Soy egresada de la carrera de Educación de la PUCMM. La educación es una disciplina íntimamente ligada a la Bibliotecología y Ciencias de la Información ya que ambas pertenecen a la rama de las humanidades. Además, la biblioteca de hoy tiene una función que va más allá del apoyo meramente instrumental, y que comprende la actividad de enseñanza y aprendizaje. Su rol es básicamente educativo, además de ser un recurso para documentarse, debe enseñar el modo de hacerlo, y no solo en lo relativo a la localización de la información, sino en su evaluación, selección, reelaboración y comunicación.

La biblioteca tiene una importante función y es formar a los usuarios en competencias informacionales. Estas se definen como un conjunto de conocimientos, habilidades y actitudes que los usuarios de este siglo necesitan para: comprender cuándo necesitan información, dónde la pueden localizar, cómo organizarla y cómo usarla de manera crítica y ética para compartir y generar conocimiento. Las bibliotecas son instituciones que contribuyen de por vida a la educación y a la formación de nuevos conocimientos.

\section{¿Cómo se organizó para poder servir con eficacia a dos Campus a la vez?}

La dirección de la biblioteca preparó y presentó proyectos de futuro a las autoridades de la PUCMM destinados a innovar y modernizar espacios, recursos de información, y servicios. También para introducir las tecnologías en todos los procesos de las bibliotecas, con el objetivo de construir un nuevo modelo, donde se integraron los diferentes elementos y características de la biblioteca universitaria del siglo 21 . 
Lo primero que se hizo fue armonizar la estructura organizativa de ambos Campus y conformar un equipo de trabajo multidisciplinario, con las destrezas y competencias requeridas. Hasta el año 1996 las Bibliotecas de la PUCMM mantuvieron un sistema de información donde el almacenamiento y la recuperación se realizaban de forma manual y de manera descentralizada. Por disposición de la rectoría, las bibliotecas se constituyeron en un Sistema con el fin de unificar criterios, procedimientos, estándares y normativas. La gestión se desarrolló bajo una sola dirección trabajando en una única plataforma tecnológica.

Se trabajó con un Plan Estratégico con ejes claves, los cuales marcaron la ruta para el trabajo: se revisó la Misión y Visión de las Bibliotecas, servicios a usuarios, infraestructuras físicas, colecciones, recursos humanos, innovación tecnológica e infraestructura digital.

\section{¿Cuáles cambios ha visto a lo largo de su gestión en el concepto y uso de la biblioteca?}

Las bibliotecas universitarias se convierten en espacios transformadores, enfrentados a grandes desafíos, producto de los cambios que se han producido en la Sociedad del Conocimiento. Uno de esos cambios es la presencia de las TICs en todas las actividades de las bibliotecas. A la par de estos cambios, las bibliotecas de la PUCMM se han transformado de tradicionales centros receptores de documentos físicos a sistemas híbridos de gestión de información. Pasaron de ser un centro pasivo a un centro de descubrimiento de nuevos conocimientos, ya que hoy, no solo se habla de recursos de información en formato físico, sino que existe un universo de información compuesto por múltiples formatos y soportes de información, sumado a la poderosa herramienta que es la Internet, la cual abre diversas posibilidades a los usuarios. Es en este contexto que el profesional de la información o bibliotecario de hoy se ha convertido en un formador de usuarios y en el motor propulsor de la producción científica universitaria.

El Sistema de Bibliotecas de la PUCMM adopta nuevas formas de gestión de la información y estilo de trabajo, obviando los procedimientos tradicionales, en vista de que las bibliotecas universitarias se abocan a nuevos retos, con el fin de dar respuesta al nuevo modelo de enseñanza universitaria. Se ha transformado y convertido en centro de recursos para el aprendizaje y la investigación. Pasó de salvaguarda de acervo hacia la oferta de servicios innovadores, para el aprendizaje, tanto tecnológico como social, conforme a las demandas de los usuarios del momento.

Las bibliotecas universitarias han tenido que adecuar los métodos y estilos de trabajo, debido a que estas instituciones contribuyen de por vida a la educación y a la formación de nuevos conocimientos y la educación de este siglo es dinámica y participativa. También se habla de la necesidad de abrir nuevas vías a través de espacios innovadores (Learning Center) para el aprendizaje tanto tecnológico como social. La biblioteca universitaria de hoy tiene que cautivar a los usuarios a través de servicios creativos. Ya los usuarios no solo se valen de los libros como tecnología principal de acceso al saber sino de nuevos instrumentos y herramientas para generar conocimientos compartidos. Por lo tanto, el compromiso es irigir las bibliotecas hacia una organización de aprendizaje y hacer de ellas espacios organizados, atractivos y flexibles, que generen, difundan y transmitan conocimientos.

\section{¿Con cuáles cambios, tanto de imagen como de funcionabilidad, ha contribuido para la mejora de este centro de investigación?}

El Sistema de Bibliotecas existente es fruto de una visión integral de lo que es una biblioteca de este siglo, en la cual se conjugan recursos y servicios de información, tanto digitales como físicos, infraestructuras tecnológicas, así como espacios flexibles y agradables. Dentro de estos cambios están:

1. Remodelación de las plantas físicas de ambos Campus.

2. Desarrollo y rediseño del Portal Web.

3. Portal enriquecido con una gran variedad de puertas de entradas y llaves de acceso a información arbitrada (videos, multimedias, entre otros).

4. Integración de las redes sociales como canales de comunicación con la comunidad de usuarios.

5. Acceso a libros extranjeros y dominicanos en formato digital.

6. Adquisición de una gran variedad importantes de bases de datos especializadas y multidisciplinarias.

7. Acceso a bases de datos especializadas de libre acceso.

8. Integración al Catálogo en Línea de Acceso Público OPAC y al Descubridor EDS con cambios significativos en la personalización de su interfaz y sus funcionalidades para servicio de los usuarios.

9. Acceso al catálogo en línea desde los dispositivos móviles de los usuarios a través de la herramienta MOPAC. 
10. Adquisición de la poderosa herramienta tecnológica EDS (Descubridor para la búsqueda simultánea de los diversos recursos de información existente en el sistema).

11. Acceso a una gran variedad de revistas y libros digitales.

12. Programa de formación con el objetivo de capacitar usuarios autosuficientes para localizar e identificar recursos de información de calidad en línea.

13. Podcast: herramienta que da visibilidad a las conferencias, seminarios, conversatorios, entre otros, impartidos por los departamentos académicos y otras instancias de la institución.

14. Apertura de cinco salas E-Learning Center para incentivar el trabajo colaborativo y la creatividad de los estudiantes, distribuidas en ambos campus.

15. Diez salas de estudio en grupo para espacios de aprendizajes.

16. Repositorio Institucional INVESTIGAREPUCMM.

17. Bibliotecario en línea para responder en forma remota a las demandas de los usuarios.

18. Biblioteca digital con biografías de dominicanos destacados.

19. Provisiones de tabletas para la lectura de contenidos digitales.

20. Digitalización de las revistas producidas por la PUCMM y que ya cesaron su publicación, como EME EME, entre otras

21. Participación en el proyecto Biblioteca Digital del Caribe.

22. Migración de los videos educativos en formato VHS ya en desuso a DVD y CD-ROM, puestos en línea al servicio de los estudiantes.

23. Servicio de Internet Wi-Fi.

24. Más de cien estaciones de trabajo con computadoras al servicio de los usuarios.

25. Renovación del mobiliario y equipo del Sistema de Bibliotecas.
26. Dos salas de estudio y de investigación especializadas al servicio de Postgrado.

27. Es la primera universidad de la República Dominicana en ofrecer un programa de Maestría en Bibliotecología y Ciencias de la Información.

Como cualquier otra organización, la biblioteca debe tener una misión clara, así como un conjunto de metas, objetivos y estrategias definidas para su desarrollo e implementación que ofrezcan resultados cuantificables y que justifique el trabajo de las bibliotecas ante los usuarios.

\section{¿Qué importancia representa una biblioteca para la educación superior?}

La biblioteca universitaria dejó de ser un cofre de tesoros para convertirse en el corazón intelectual de la universidad. Ocupa un lugar central y básico, es un recurso que atiende y sirve a toda la función de una universidad, enfocado en la enseñanza, la investigación y la creación de nuevos conocimientos y la transmisión para la posteridad de la ciencia y la cultura.

La UNESCO afirma que el papel principal de la biblioteca universitaria es el educativo, por lo tanto, no debe verse como mero depósito de libros con salas de lectura, sino como un espacio dinámico al servicio de la educación superior. La biblioteca universitaria juega un papel indispensable en sintonía con la misión de la universidad ayudando a los estudiantes en la localización del conocimiento y del saber con rigor científico.

El bibliotecario de hoy no debe ocuparse tanto por las tareas rutinarias, sino que su rol principal es la promoción del estudio y del conocimiento, así como la formación a profesores, estudiantes e investigadores a fin de asegurar la exactitud y credibilidad, exploración y verificación de las informaciones que se suministran.

La biblioteca está muy relacionada con los métodos docentes que la universidad aplica. Si el estudiante es un ente pasivo, que aprende asistiendo a clase y tomando apuntes, la biblioteca tiene poca importancia porque los estudiantes la utilizan solo como sala para estudiar. Sin lugar a dudas, los nuevos métodos de enseñanza aprendizaje contribuyen a una mayor integración y valor de la biblioteca universitaria debido a que el nuevo método permite la participación activa de los estudiantes $y$, por lo tanto, requieren una mayor utilización de los recursos y servicios de la biblioteca.

Las bibliotecas universitarias tienen una función que va más allá del apoyo meramente instrumental a la actividad de enseñanza y aprendizaje. Una función 
«intrínsecamente» educativa, además de ser un recurso para documentarse, deben enseñar el modo de hacerlo, y no solo en lo relativo a la localización de la información, sino en su evaluación, selección, reelaboración, uso y comunicación. Esto es, contribuir a la formación de la competencia informacional, que incluye tanto aspectos procedimentales como conceptuales y éticos, y es básica para un aprendizaje activo, constructivo y contextualizado.

La importancia de la biblioteca en la universidad es tal que se constituye en uno de los principales indicadores de calidad de la institución y pilar para la acreditación de las universidades.

\section{¿En qué posición se encuentra el Sistema de Bibliotecas de la PUCMM con respecto a los demás?}

El Sistema de Bibliotecas de la PUCMM tiene grandes fortalezas. Es la primera Biblioteca universitaria privada del país, ha servido de modelo para el desarrollo de otras bibliotecas dominicanas universitarias, ya que fue diseñada y construida por expertos, de acuerdo a los más altos estándares internacionales de calidad. Se distingue por sus ricas y únicas colecciones. Por la cantidad, calidad y diversidad de sus recursos de información, consultados por, entre otros, estadistas, ministros, funcionarios del gobierno, investigadores internacionales y nacionales especialistas en diversas ramas de la ciencia. Se destaca también por la cuidadosa y constante selección de sus adquisiciones, como por su sistema de organización. Su personal está capacitado y formado en Bibliotecología, Ciencias de la Información y ciencias afines. Cuenta con una de las mejores plataformas tecnológicas de calidad mundial.

El Banco Mundial la seleccionó como única depositaria en el país de una producción bibliográfica internacional con implementación de herramientas tecnológicas innovadoras al servicio de los usuarios.

El Catálogo Universidad Católica Madre y Maestra (PUCMM), fue valorado en un medio internacional de alto prestigio como ejemplo de innovación, por haber realizado cambios significativos en la personalización de su interfaz y funcionalidades del OPAC. La Comunidad Baratz de España, presenta el OPAC de la PUCMM como ejemplo de innovación continua y buenas prácticas que sirven de ejemplo para mejorar los OPAC de otras bibliotecas a nivel nacional e internacional.

El Sistema de Bibliotecas ha recibido numerosos premios y distinciones de la Asociación de Bibliotecas Universitarias, de Investigación e Institucionales del Caribe (ACURIL):
- Premio "Albertina Pérez de Rosa", ACURIL 2018, Santo Domingo, República Dominicana.

- Premio a la "Investigación y Creatividad en el Campo de la Información y el Conocimiento", ACURIL 2017, San Juan, Puerto Rico.

- Premio Profesional de la Información del Caribe y Medalla al Mérito de Excelencia, ACURIL 2015 Suriname.

- Leadership in the Development of Indispensable Research Solutions that Connect People and Information ProQuest Award, ACURIL 2014 Nassau, Bahamas".

- Top Finalist del premio ProQuest Leadership in the Development of Research Solutions that Connect People and Information.

- Medalla Acurileana de Excelencia como la Caribbean Information Professional of the Year Award y con la placa EBSCO Information Services, The Award for Leadership.

- Premio a la Excelencia en Programas de Conservación y Preservación de Colecciones del Caribe 2003 y por el Rescate de la Documentación y la Creación de Colecciones Especiales de Investigación en la República Dominicana.

- Premio a la Excelencia en el desarrollo e implementación de servicios bibliotecarios efectivos e innovadores en el Caribe con la aplicación de tecnologías de información.

- Premio ACURIL PUBLIC RELATIONS AWARD 1995.

El Sistema de Bibliotecas está afiliado a organizaciones de prestigio como:

$\diamond \quad$ Miembro fundador de la Asociación de Bibliotecas Universitarias Dominicanas (ABUD)

$\diamond$ Asociación de Bibliotecas Universitarias y de Investigación e Institucionales del Caribe (ACURIL).

$\diamond \quad$ OCLC (Online Computer Library Center)

$\diamond$ Red Latinoamericana de Información y Documentación en Educación REDUC

$\diamond \quad$ Miembro del Comité Consultivo Biblioteca Virtual en Salud (BVS-RD) 
$\diamond$ Seminario de adquisiciones de materiales Latinoamericanos para Bibliotecas (SALALM) Mundial

$\diamond \quad$ Miembro del Comité Consultivo de la Biblioteca Digital del Caribe

\section{¿Cómo debe ir asumiendo la biblioteca el cambio del libro físico al virtual?}

La incorporación de los libros electrónicos a las bibliotecas implica una serie de cambios en los procesos de gestión de la información. Investigaciones recientes vaticinan la coexistencia del libro físico con el libro digital. Este último complementa al libro en soporte papel en las bibliotecas, en vista de que hay lectores fieles al soporte impreso y nuevos lectores como los milennials y la generación Z, que no saben vivir sin su lector de libros electrónicos. La tendencia es que las bibliotecas deben formar colecciones hibridas, es decir, en formato impreso y en digital. Los usuarios de hoy utilizan más servicios en línea y hacen mucho uso de la lectura digital.

En la actualidad se recomienda sustituir espacios destinados a colecciones por espacios destinados a los usuarios, esto beneficia mucho porque los libros digitales pueden estar en un disco duro o virtualmente almacenados, sin ocupar espacio físico. Otra de las ventajas de este tipo de soporte es la facilidad de localización y que el préstamo se puede realizar sin importar el día y horario.

Sin embargo, un asunto a considerar es que el libro digital no pertenece a la biblioteca, lo que se paga es una licencia de acceso limitado por número de usuarios al contenido, por un tiempo determinado. Otra modalidad puede ser la compra a perpetuidad de estos recursos.

Algunos autores predicen que si la biblioteca continúa centrándose en adquirir recursos de información en soporte papel corren el riesgo de no justificarse ante la sociedad, ya que la forma de leer no cambia, lo que cambia es el soporte, por lo que la biblioteca debe proveer información en diferentes formatos, tanto impreso como electrónico. Las bibliotecas deben saber adaptarse a los cambios sin perder la visión de acceso a la información.

¿En qué otras áreas de la Universidad se ha desempeñado o ha colaborado y sigue colaborando?

Desde 1975 al 1986 inicié como auxiliar de la Biblioteca en el Campus Central y luego pasé a ser encargada de servicio al público.

En el año 1986 me nombraron como Directora de Registro del Campus Santo Tomás de Aquino. En la actualidad me desempeño como docente y coordinadora del primer programa de Maestría en Bibliotecología y Ciencias de la información impartido en la República Dominicana, con el fin de contribuir en la formación de los profesionales en este campo que tanto necesita el país. Además, a lo largo de mi gestión nos hemos ocupado de la impartición permanente de Diplomados y cursos para formar a los auxiliares, tanto del Sistema de Bibliotecas de PUCMM como los que laboran en las bibliotecas del país.

¿Qué recomendaciones puede dar a los docentes para que saquen mayor provecho a esta fuente permanente del desarrollo científico?

La primera recomendación es recordar a los docentes el papel que juega la biblioteca como fuente confiable de información. La biblioteca es la que asegura la exactitud y credibilidad de la información que se está suministrando a través de la red. El docente debe ser un aliado de la biblioteca, deberá interactuar con el personal bibliotecario para conocer la diversidad de recursos disponibles en la biblioteca y así satisfacer las demandas de información de su disciplina. Asimismo, deberá direccionar y concientizar a sus alumnos sobre la importancia de utilizar estos recursos para el desarrollo de sus trabajos de investigación. El docente debe considerar a la biblioteca como el principal apoyo académico en el suministro de información y conocimiento, insumos básicos para el proceso de aprendizaje que realiza el estudiante en su papel de aprendedor y en el uso ético de la información. Por lo cual, el docente debe diseñar sus estrategias de enseñanza vinculadas al uso de los recursos de información existentes en la biblioteca.

Es perentorio en este momento que estamos viviendo que la biblioteca esté integrada al proyecto educativo de la universidad y al proceso de enseñanza aprendizaje como herramienta básica para la formación de sus alumnos. 